\title{
Confiabilidad interpretativa sobre la construcción científica de premisas en la redacción problemática de los manuscritos
}

Interpretative reliability on the scientific construction of premises in the problematic writing of manuscripts

Recibido: marzo 05 de 2019 | Revisado: mayo 13 de 2019 | Aceptado: junio 17 de 2019

\author{
Ramiro M. Yallico Calmett ${ }^{\text {IA }}$ \\ George Argota Pérez ${ }^{2, *}$ \\ Patricia E. Pardo Angulo ${ }^{\mathrm{iB}}$ \\ Flor A. Lavanda Reyes ${ }^{\text {IC }}$ \\ Henry G. Guevara Garibay ${ }^{\text {ID }}$ \\ José C. Rodríguez CHACóN ${ }^{\text {ID }}$
}

\begin{abstract}
Resumen
El propósito de este trabajo fue evaluar la confiabilidad interpretativa sobre la construcción científica de premisas en la redacción problemática de los manuscritos. Se analizaron 22 propuestas de manuscritos: 14 presentadas como trabajos en extenso durante la participación de congresos y ocho para su publicación en revistas indexadas desde agosto de 2018 hasta febrero de 2019. Se seleccionaron 10 docentes evaluadores para analizar la calidad introductoria de cada propuesta donde se midieron cinco estructuras metodológicas: análisis de contexto, antecedentes, vacío del conocimiento, objetivos y fundamentación estableciéndose un criterio de análisis según escala tipo Likert. Se comparó los resultados de las calificaciones mediante un ANOVA. La confiabilidad de los resultados indicados fue mediante alfa de Cronbach. El promedio otorgado por los docentes fue de 17,9 (total: 20) señalando que la evaluación sobre las propuestas fue muy aceptada $(3,58$; total cuatro puntos). No hubo diferencias estadísticas significativas ( $\mathrm{p}$ $=.6034)$ con relación a las evaluaciones docentes. Sin embargo, al realizar una estadística t para una muestra (grados de libertad: 9) se observó que, el valor $\mathrm{p}=0$ donde la $\mathrm{H} 1$ expresó que la confiabilidad interpretativa de docentes evaluadores sobre la construcción de premisas en los manuscritos científicos es de calidad no aceptada lo cual, mostró contradicción en lo interpretado por los docentes siendo corroborado mediante la confiabilidad hallada por el alfa de Cronbach (0,5: moderada). Se concluye que, se requiere aumentar la preparación metodológica en docentes evaluadores para mejorar su interpretación sobre la construcción de premisas en los manuscritos científicos.
\end{abstract}

Palabras clave: comunicación científica, interpretación metodológica, problematización científica, redacción

\begin{abstract}
The purpose of the work was to evaluate the interpretative reliability of the scientific construction of premises in the problematic writing of manuscripts. 22 manuscripts proposals were analyzed: 14 submitted as extensive work during the participation of congresses and eight for publication in indexed journals from August 2018 to February 2019.10 evaluator teachers were selected to analyze the introductory quality
\end{abstract}

\footnotetext{
(C) Los autores. Este artículo es publicado por la Revista Campus de la Facultad de Ingeniería y Arquitectura de la Universidad de San Martín de Porres. Este artículo se distribuye en los términos de la Licencia Creative Commons Atribución No-comercial - Compartir-Igual 4.0 Internacional (https://creativecommons.org/licenses/ CC-BY), que permite el uso no comercial, distribución y reproducción en cualquier medio siempre que la obra original sea debidamente citada. Para uso comercial contactar a: revistacampus@usmp.pe.
} 
of each proposal where five methodological structures were measured: context analysis, background, knowledge gap, objectives and foundation establishing a criteria according to Likert scale. Grade results were compared using an ANOVA. The reliability of the indicated results was by Cronbach alpha. The average given by teachers was 17.9 (total: 20) noting that the assessment of the proposals was very accepted (3.58; total four points). There were no significant statistical differences ( $p$.6034) in relation to teacher assessments. However, when performing a t-statistic for a sample (degrees of freedom: 9) it was observed that, the p-value .000 where $\mathrm{H} 1$ expressed that, the interpretative reliability of teaching evaluators on the construction of premises in scientific manuscripts is of unaccepted quality, which showed contradiction in what teachers interpreted being corroborated by the reliability found by Cronbach's alpha ( 0.5 : moderate). It was concluded that increasing methodological preparation in teaching evaluators is required to improve their interpretation of the construction of premises in scientific manuscripts.

Key words: scientific communication, methodological interpretation, scientific problematization, drafting

\section{Introducción}

La estructura de los trabajos de investigación y sus descripciones representan la funcionalidad sobre lo que se desea comunicar (McDonnell, 2017). No solo basta escribir, pues luego se requiere que sea aceptado para su publicación (Bajwa \&Sawhney, 2016). El resultado de esta publicación, constituye un indicador de mérito para el investigador científico quien reconoce que, se aprende a escribir mediante el reto y entrenamiento formal de dicha práctica (Auvinen, 2015). Sin embargo, redactar de manera comprensible y aceptable, no es tarea fácil debido a, la propia subjetividad que genera apreciar lo que se escribe, pero ajeno al interés de otros. Las publicaciones en sí mismas, persiguen un fin de potenciación, perspectivas o justificación (Pierson, 2004; Guyatt \& Brian, 2006; Driscoll \& Aquilina, 2011; El-Serag, 2012). Todo manuscrito científico al recepcionarse, tiene dos ópticas: juzgarse por el mérito científico o rechazarse por inconsistencia incluyendo, la desordenada escritura (Powell, 2010; Whitehouse, 2013).

Antes de redactar un manuscrito científico es fundamental identificar la revista adecuada donde exista probabilidad de ser el manuscrito aceptado, así como también de entender la originalidad de la propuesta, transparencia y relevancia de los resultados obtenidos (Sengupta et al., 2014). Marco \& Schmidt (2004) y Menezes et al., (2006) indican que, lo primordial antes de la escritura radica en revisar exhaustivamente la información disponible en la literatura para reconocer, la necesidad sobre un posible conocimiento a comunicar donde identificar el tratamiento de los datos resultará el imperativo sobre la intensión a comunicar.

Dentro de las principales dificultades en los manuscritos científicos está la comprensión sobre qué información científica se desea comunicar según la 
fuente de citación desde su concepción de origen y alcance cognoscitivo hasta la validación de los hallazgos, así como, el criterio ofrecido por parte posibles evaluadores sobre las propuestas a publicar en revistas científicas. El propósito del presente trabajo de investigación, fue evaluar la confiabilidad interpretativa sobre la construcción científica de premisas en la redacción problemática de los manuscritos.

\section{Materiales y métodos}

\section{Objeto de investigación y periodo de estudio}

El estudio se realizó en propuestas de manuscritos quefueron presentados como trabajo en extenso para la participación de congresos y publicaciones en revistas Tabla 1.

Estructuras metodológicas / escala tipo Likert

\begin{tabular}{|c|c|c|c|}
\hline \multicolumn{4}{|c|}{ Análisis de contexto } \\
\hline Muy alto & Alto & Bajo & Muy bajo \\
\hline 4 & 3 & 2 & 1 \\
\hline \multicolumn{4}{|c|}{ Antecedentes } \\
\hline Muy alto & Alto & Bajo & Muy bajo \\
\hline 4 & 3 & 2 & 1 \\
\hline \multicolumn{4}{|c|}{ Vacío del conocimiento } \\
\hline Muy alto & Alto & Bajo & Muy bajo \\
\hline 4 & 3 & 2 & 1 \\
\hline \multicolumn{4}{|c|}{ Objetivos } \\
\hline Muy alto & Alto & Bajo & Muy bajo \\
\hline 4 & 3 & 2 & 1 \\
\hline \multicolumn{4}{|c|}{ Fundamentación } \\
\hline Muy alto & Alto & Bajo & Muy bajo \\
\hline 4 & 3 & 2 & 1 \\
\hline
\end{tabular}

Se planteó como hipótesis lo siguiente:

- Ho: La confiabilidad interpretativa de docentes evaluadores sobre la construcción de premisas en los manuscritos científicos es de calidad aceptada. científicas indexadas desde agosto de 2018 hasta febrero de 2019.

\section{Selección de muestra}

De 22 propuestas (14: trabajos para congresos; ocho como artículos) fueron seleccionados mediante un muestreo probabilístico aleatorio.

\section{Análisis de variables}

Se analizó, únicamente la introducción con relación a la propuesta del título donde se evaluó cinco estructuras metodológicas: análisis de contexto: I, antecedentes: II, vacío del conocimiento: III, objetivos: IV y fundamentación: V (Argota, 2018). Las estructuras se caracterizaron mediante una escala Likert, asignándose valores numéricos (Tabla 1). 
Para validar las estructuras se analizó la consistencia (fiabilidad) mediante la aplicación del coeficiente alfa de Cronbach (1951), utilizando el método de la varianza de los ítems:

$$
\alpha=\mathrm{K} / \mathrm{K}-1\left[1-\sum \mathrm{V} 1 / \mathrm{Vt}\right]
$$

Donde: $\alpha=$ alfa de Cronbach; $\mathrm{K}=$ número de items; $\mathrm{V} 1$ = varianza de cada items; $\mathrm{Vt}=$ varianza total.

\section{Análisis estadístico de los datos}

Se utilizó el programa estadístico Epidat 4.2 (versión libre) donde se describió el promedio, el análisis de varianza, contraste múltiple de rangos (Tukey HSD) y el contraste de hipótesis mediante el análisis t para una muestra (promedio de calificación interpretativade los docentes considerando significativos los resultados cuando $\mathrm{p}<.05$.

\section{Resultados}

La Tabla 2 muestra, la calificación numérica para las cinco estructuras metodológicas según el promedio otorgado de los docentes evaluadores con relación a las siete propuestas científicas.

Tabla 2

Promedio / escala tipo Likert

\begin{tabular}{|c|c|c|c|c|c|}
\hline Docente & $\begin{array}{c}\text { Análisis de } \\
\text { contexto }\end{array}$ & Antecedentes & $\begin{array}{c}\text { Vacío del } \\
\text { conocimiento }\end{array}$ & Objetivos & Fundamentación \\
\hline 1 & 1 & 3 & 4 & 4 & 4 \\
\hline 2 & 4 & 4 & 2 & 3 & 4 \\
\hline 3 & 4 & 4 & 3 & 4 & 3 \\
\hline 4 & 3 & 4 & 4 & 4 & 4 \\
\hline 5 & 4 & 4 & 4 & 4 & 3 \\
\hline 6 & 4 & 4 & 4 & 3 & 4 \\
\hline 7 & 2 & 3 & 4 & 4 & 4 \\
\hline 8 & 4 & 4 & 3 & 4 & 4 \\
\hline 9 & 3 & 3 & 4 & 3 & 3 \\
\hline 10 & 4 & 4 & 3 & 4 & 4 \\
\hline Promedio & 3,3 & 3,7 & 3,5 & 3,7 & 3,7 \\
\hline Items & \multicolumn{5}{|c|}{ Criterio } \\
\hline & Muy alto & \multicolumn{2}{|c|}{ Alto } & Bajo & Muy bajo \\
\hline & 4 & \multirow{2}{*}{\multicolumn{2}{|c|}{$\begin{array}{c}3 \\
3,3\end{array}$}} & 2 & 1 \\
\hline 1 & & & & & \\
\hline 2 & 3,7 & & & & \\
\hline 3 & 3,5 & & & & \\
\hline 4 & 3,7 & & & & \\
\hline 5 & 3,7 & & & & \\
\hline
\end{tabular}

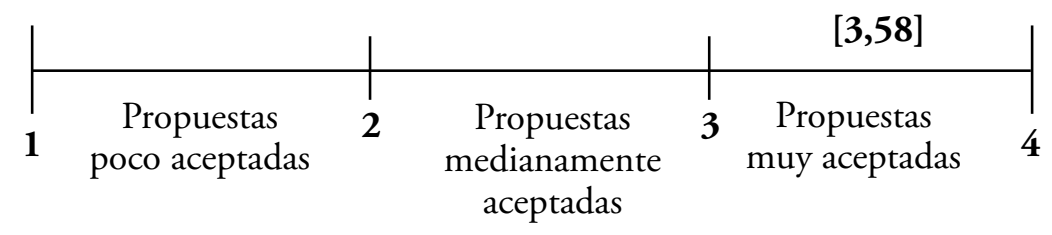


La Tabla 3 muestra el análisis de varianza y contraste múltiple de rangos (prueba Tukey HSD) según el criterio de puntuación de los docentes evaluadores con relación a la construcción de premisas en la redacción problemática de los artículos como valor de utilidad por citación. No se encontró, diferencias estadísticas significativas $(\mathrm{p}=.6034)$ con relación a las evaluaciones docentes.

Tabla 3

Análisis de varianza

\begin{tabular}{|c|c|c|c|c|c|}
\hline FV & SC & $\mathrm{gl}$ & $\mathrm{CM}$ & $\mathrm{F}$ & $\mathrm{P}$ \\
\hline Entre grupos & 1,28 & 4 & 0,32 & 0,69 & .6034 \\
\hline Intra grupos & 20,9 & 45 & 0,464444 & & \\
\hline Total (Corr.) & 22,18 & 49 & & & \\
\hline $\begin{array}{l}\text { Estructuras } \\
\text { metodológicas }\end{array}$ & Promedio & & Homogéneos & & \\
\hline Análisis de contexto & 3,3 & & $\mathrm{X}$ & & \\
\hline Antecedentes & 3,5 & & $\mathrm{X}$ & & \\
\hline Vacío del conocimiento & 3,7 & & $\mathrm{X}$ & & \\
\hline Objetivos & 3,7 & & $\mathrm{X}$ & & \\
\hline Fundamentación & 3,7 & & $\mathrm{X}$ & & \\
\hline
\end{tabular}

La Figura 1 muestra, la distribución $\mathrm{t}_{\text {tab }}$ promedio de calificaciones docentes evaluadores con relación a la construcción de premisas en la redacción problemática de los artículos como valor de utilidad por citación donde la estadística básica para t de 1 muestra refirió lo siguiente: Hipótesis nula $-\mathrm{H}_{0}: \mu=15$; Hipótesis alterna $-\mathrm{H}_{1}: \mu>15$; valor $\mathrm{T}=7,14$, valor $\mathrm{p}=.000$.

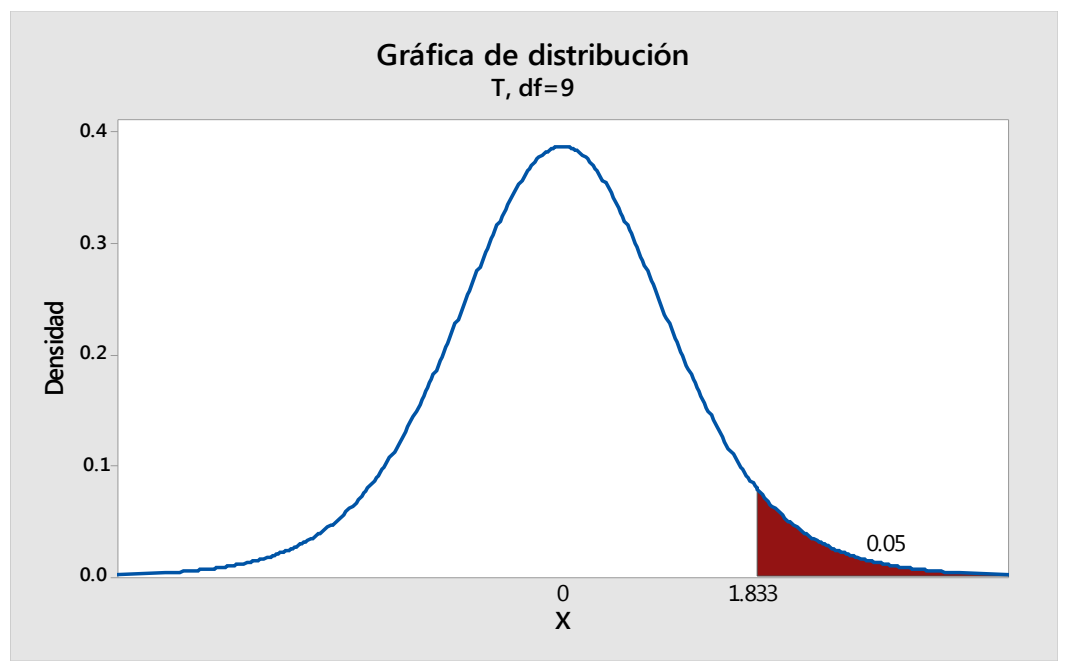

Figura 1. Distribución $\mathrm{t}$ tab / promedio de calificaciones docentes evaluadores.

La Tabla 4 muestra la confiabilidad de los datos medidos según el alfa de Cronbach para los docentes evaluadores con relación a la construcción de premisas en la redacción problemática de los artículos como valor de utilidad por citación. 
Tabla 4

Confiabilidad de los datos / alfa de Cronbach / V = varianza

\begin{tabular}{cccccccc}
\hline Docente & $\begin{array}{c}\text { Análisis de } \\
\text { contexto }\end{array}$ & Antecedentes & $\begin{array}{c}\text { Vacío del } \\
\text { conocimiento }\end{array}$ & Objetivos & Fundamentación & $\sum \mathrm{V}$ & $\mathrm{Vt}$ \\
\hline 10 & 1,12 & 0,23 & 0,5 & 0,23 & 0,23 & 2,31 & 1,65 \\
\hline Indicador & Valor & & & & & & \\
\cline { 1 - 2 } $\mathrm{K}$ & 5 & & & & & & \\
$\mathrm{~V} 1$ & 2,46 & & & & & & \\
$\mathrm{Vt}$ & 1,65 & & & & & & \\
\cline { 1 - 2 }
\end{tabular}

Por tanto, $\alpha=0,5$.

La Figura 2 muestra la asignación de de premisas en la redacción problemática puntuación moderada según el alfa de de los artículos como valor de utilidad Cronbach con relación a la construcción por citación.

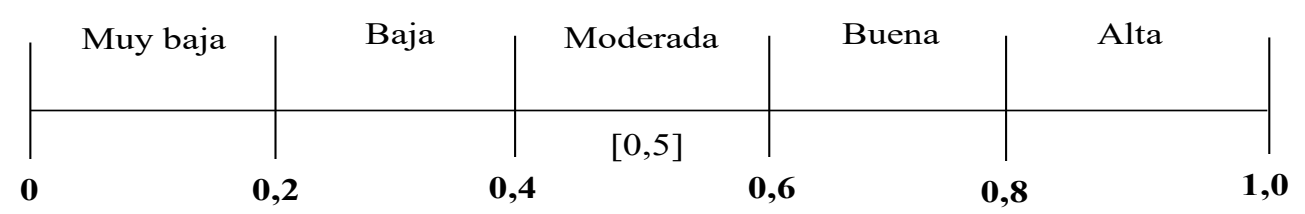

Figura 2. Análisis de la consistencia interpretativa / alfa de Cronbach.

\section{Discusión}

De los resultados mostrados en la sección anterior se observó que el promedio concedido por los docentes evaluadores fue de 17,9 puntos (de 20 puntos) lo cual resultó luego, en aceptadas las propuestas de los manuscritos debido al, promedio de 3,58 puntos (total: 4). A pesar que, no se encontró diferencias en las evaluaciones de los docentes, la estadística t para una muestra (grados de libertad: 9) registró, valor $\mathrm{p}=.000$ donde se aceptó la $\mathrm{H} 1$ (alterna)quien expresó que, la confiabilidad interpretativa de docentes evaluadores sobre la construcción de premisas en los manuscritos científicos es de calidad no aceptada, mostrándose contradicción en lo anteriormente interpretado por los docentes y luego, corroborado mediante la confiabilidad hallada por el alfa de Cronbach.

Cualquier análisis sobre las propuestas de manuscritos científicos, es una medida individual de la posible publicación (Belcher, Barron \&Yang, 2016) de modo que, resulta necesario en términos formativos valorar la calidad sobre lo que se propone, pues ello significaría un reflejo sobre la posible redacción personal, pues no basta el desarrollo propiamente de investigación sino, en asegurar la calidad intermedia y final de resultado a presentar (Lin \& Evans, 2012; Lillis \& Curry, 2015). En el análisis de un manuscrito, no solo se evalúa el grado de colaboración del mismo, además, se mide la calidad en la escritura (Bremner et al., 2014; Van Steendam, 2016).

\section{Conclusiones}

Se concluyó que se requiere aumentar la preparación metodológica en docentes evaluadores para mejorar su interpretación sobre la construcción de premisas en los manuscritos científicos. 


\section{Referencias}

Argota, P.G. (2018). Problema científico. Biotempo; 15(2), 217-229. https:// doi.org/10.31381/biotempo. v15i22060

Auvinen, A. (2015). How do I write a scientific article-advice to a young researcher. Duodecim; 131(16), 1460-1466.

Bajwa, S.J. \& Sawhney, C. (2016). Preparing manuscript: scientific writing for publication. Indian J. Anaesth. 60(9), 674-678. https://doi.org/10.4103/00195049.190625

Belcher, D.D., Barron, S.F.H. \& Yang, H.S. (2016). English for professional academic purposes. In: Shaw, P., Hyland, K. (Eds.). The Routledge handbook of English for Academic Purposes. Routledge, Abingdon, Oxon; 502-514.

Bremner, S., Pierson, S.A., Jones, R. \& Bhatia, V. (2014). Task design and interaction in collaborative writing: The students' story. Bus. Profess. Commun. Quart; 77(2), $\quad$ 150-168.https://doi. org/10.1177/2329490613514598

Driscoll,J. \&Aquilina, R. (2011). Writing for publication: a pratical six-step approach. Int $J$ Orthop Trauma Nurs, 15, 41-48.https://doi. org/10.1016/j.ijotn.2010.05.001

El-Serag, H.B. (2012). Writing and publishing scientific papers. Gastroenterology; 142 , $197-$ 200. https://doi.org/10.1053/j. gastro.2011.12.021
Guyatt, G.H. \& Brian, H.R. (2006). Preparing reportsforpublicationand responding to reviewers' comments. J Clin Epidemiol; 59, 900-906. https://doi.org/10.1016/j. jclinepi.2006.05.004

Lillis, T. \& Curry, M.J. (2015). The politics of English, language and uptake. The case of international academic journal article reviews. AILA Rev; 28, 127-150.https:// doi.org/10.1075/aila.28.06lil

Lin, L. \& Evans, S. (2012). Structural patterns in empirical research articles: A crossdisciplinary study. Engl. Specif. Purp; 31, 150-160.

Marco, C.A. \& Schmidt, T.A. (2004). Who wrote this paper. Basics of authorship and ethical issues? Acad. Emerg. Med; 11, 76-77.

McDonnell,J.J.(2017).Paperwritinggone Hollywood. Science; 355(6320), 102.https://doi.org/10.1126/ science.355.6320.102

Pierson, D.J. (2004). The top 10 reasons why manuscripts are not accepted for publication. Respir Care; 49, 1246-1252.http://www.rcjournal. com/contents/10.04/10.04.1246. pdf

Powell, K. (2010). Publish like a pro. Nature; 467, 873-875.

Sengupta, S., Shukla, D., Ramulu, P., Natarajan, S. \& Biswas, J. (2014). Publish or perish: the art of scientific writing. Indian J. Ophthalmol; 
Ramiro M. Yallico Calmett - George Argota Pérez - Patricia E. Pardo Angulo - Flor A. Lavanda Reyes - Henry G. Guevara Garibay - José C. Rodríguez Chacón

62(11), 1089-1093.https://doi. Whitehouse, S. (2013). How to org/10.4103/0301-4738.146760

Van Steendam, E. (2016). Editorial: write for publication in medical journals. Transl Res; 162, 270-273. forms of collaboration in writing. https://doi.org/10.4103/2230J. Writing Res; 8(2), 183-204. 8210.102988 https://doi.org/10.17239/jowr2016.08.02.01 